Revue d'histoire du XIXe siècle

Société d'histoire de la révolution de 1848 et des

révolutions du XIXe siècle

49 | 2014

1814-1815. Expériences de la discontinuité

\title{
La magistrature française face aux deux Restaurations (1814-1815)
}

The French Judiciary in front of the two Restorations (1814-1815)

Die französische Justiz im Zeichen der Restauration (1814-1815)

\section{Olivier Tort}

\section{(2) OpenEdition}

\section{Journals}

Édition électronique

URL : http://journals.openedition.org/rh19/4749

DOI : $10.4000 /$ rh 19.4749

ISSN : $1777-5329$

Éditeur

La Société de 1848

\section{Édition imprimée}

Date de publication : 1 décembre 2014

Pagination : 93-107

ISSN : 1265-1354

Référence électronique

Olivier Tort, "La magistrature française face aux deux Restaurations (1814-1815) », Revue d'histoire du XIXe siècle [En ligne], 49 | 2014, mis en ligne le 01 décembre 2014, consulté le 19 avril 2019. URL: http://journals.openedition.org/rh19/4749; DOI : 10.4000/rh19.4749 


\section{OLIVIER TORT}

\section{La magistrature française face aux deux Restaurations (1814-1815)}

Après la vaste réorganisation de 1808-1810, la forte instabilité institutionnelle des deux années 1814 et 1815 place à nouveau la magistrature dans une situation délicate. Elle réactive en son sein des oppositions politiques internes jusque-là mises en sourdine par l'autoritarisme du régime impérial. La continuité - ce gage supposé d'une justice sereine - se trouve interrompue et fait place à des chocs répétés, qui ramènent, nolens volens, à des périodes de troubles antérieures et notamment aux clivages des années révolutionnaires. Dès les débuts de la Première Restauration, les déclarations, les adresses, le compte rendu des audiences princières et royales font apparaitre de subtiles différences d'attitude selon les juridictions, qui sont elles-mêmes aiguisées par l'accueil plus ou moins chaleureux des nouvelles autorités politiques. De vives controverses médiatiques et parlementaires donnent l'occasion de débattre âprement de l'indépendance de la justice et des devoirs d'un bon magistrat.

La crainte d'une épuration, qui avait paru écartée lors du premier retour de Louis XVIII, se profile avant même les Cent-Jours. Cette épée de Damoclès explique le caractère relativement massif du ralliement des magistrats à l'Empereur lors de son retour, puis, à l'inverse, les stratégies de dissimulation zélée qui se déploient sous la seconde Restauration pour éviter une éviction qui menace, selon les ressorts territoriaux, jusqu'en 1821.

La réflexion sera ici limitée à l'ordre judiciaire stricto sensu, sans inclure les juridictions administratives (Conseil d'État) et financières (Cour des comptes, objet d'un important débat en novembre 1815). Seront laissées également de côté les juridictions d'exception que sont les cours prévôtales, rétablies par la loi du 27 décembre 1815 et n'entrant en application qu'à partir de 1816, hors du champ chronologique retenu. Malgré ces restrictions, on peut voir à quel point les deux années de restauration chaotique du trône des Bourbons ont constitué, en elles-mêmes, un épisode décisif des rapports toujours délicats entre le pouvoir politique et la magistrature.

Car avant que ne survienne le temps des consolidations et d'une refondation qui se veut réfléchie, cette double année 1814-1815 est celle de tem- 
poralités beaucoup plus hachées pour les différents acteurs de la justice, où l'individuel reprend souvent son autonomie par rapport au collectif, dans des stratégies de survie professionnelle menées, en grande partie, à l'aveugle. À l'incertitude inquiète des premiers mois, où acteurs politiques royalistes et magistrats issus de la Révolution et de l'Empire se regardent en chien de faïence, succède une accélération liée aux Cent-Jours; elle oblige chacun à se positionner et à prendre ses responsabilités, par calcul opportuniste de "girouette» ${ }^{1}$ ou en se conformant avec sincérité à une idéologie familiale.

Ces choix effectués sous le sceau du provisoire et sans visibilité d'avenir permettent au pouvoir royaliste rétabli de trancher, en apparence, le nœud gordien par le choix d'une épuration méthodique, dépassant les sanctions au cas par cas. Précisément, il apparaît nécessaire de reconsidérer le tempo et l'ampleur de cette sélection punitive, qui fait partie depuis deux siècles des poncifs de l'historiographie ${ }^{2}$ et montre des magistrats victimes et parfois acteurs de la "Terreur blanche ${ }^{3}$. Il ressort d'un examen minutieux qu'il s'est agi, non d'une déferlante vengeresse, mais, bien plutôt, d'un processus minutieusement contrôlé, où les principaux acteurs se sont efforcés, envers et contre tout, de maîtriser le temps et d'éviter un emballement destructeur.

\section{LE TEMPS DES ILLUSIONS ET DES FAUX-SEMBLANTS}

La chute de l'Empire fin mars 1814 ouvre, pour les agents publics, une ère d'incertitude, qui nourrit tout à la fois espoirs et inquiétudes. À cette date, les promesses de stabilisation du personnel judiciaire faites par Bonaparte lors de son accession au pouvoir, quinze ans avant, n'apparaissent plus crédibles aux yeux des magistrats. Le principe d'inamovibilité de la magistrature garanti dans la constitution de l'an VIII (article 68) a été profondément malmené, à deux reprises, sous l'Empire : en instituant une période probatoire de cinq ans, le sénatus-consulte du 12 octobre 1807 et ses décrets d'application du 24 mars 1808 ont permis à Napoléon d'évincer 162 magistrats du siège ceux du parquet étant, par définition à l'époque, révocables à loisir; puis la fusion des cours d'appel et des cours criminelles en cours impériales, mise en œuvre par la loi du 20 avril 1810, a fait discrètement révoquer encore beaucoup d'autres magistrats, toujours théoriquement inamovibles ${ }^{4}$.

1. Cf. Pierre Serna, La République des girouettes : 1789-1815... et au-delà : une anomalie politique. La France de l'extrême-centre, Seyssel, Champ Vallon, 2005.

2. Dans L'histoire de la justice française de la Révolution à nos jours, trois décennies de recherche (Paris, PUF, 2001, p. 221), Jean-Claude Farcy relève le caractère "répétitif " des études sur l'épuration judiciaire.

3. Cf. Laurent Nagy, D’une Terreur à l'autre. Théories du complot et nostalgie de l'Empire, 18151816, collection "Chroniques", Paris, Vendémiaire, 2012, p. 30-31.

4. Cf. Marcel Rousselet, Histoire de la magistrature française des origines à nos jours, Paris, Plon, 1957, vol. 2, p. 166; Jean-Pierre Royer, Histoire de la justice en France, Paris, Presses universitaires de France, $3^{e}$ éd. Augmentée, 2001, p. 485-488. 
Cette démarche d'une épuration qui ne dit pas son nom aboutit donc, dès avant 1814, à une contraction des effectifs judiciaires nationaux, qui s'accentue encore au rythme des défaites militaires napoléoniennes de la campagne d'Allemagne (1813) puis à l'issue de la signature du premier traité de Paris (30 mai 1814) : les ressorts judiciaires de Bruxelles, Florence, Gênes, Hambourg, La Haye, Liège, Rome, Trêves et Turin cessent d'être français. Si les magistrats autochtones de ces régions, très majoritaires, peuvent parfois espérer, selon les cas de figure, être maintenus dans le cadre de leurs nouveaux États d'appartenance en reniant leur francophilie, la situation de la minorité de juges d'origine française est encore plus délicate et repose sur l'éventuelle recomposition des juridictions françaises, et donc sur l'espoir d'une épuration qui leur permette de se réinsérer au détriment d'autres collègues 5 . Au printemps 1814, au sein de ces frontières rétrécies, il y a donc à peine 5500 magistrats selon nos estimations, soit environ 1500 magistrats de moins que ceux qui seront dénombrés treize ans plus tard, sous l'ère Villèle (1827), lors de la confection du premier registre matricule général de la profession ${ }^{6}$. Les trois quarts du corps relèvent des tribunaux de première instance : ces magistrats, allant des juges suppléants au président de tribunal, forment une sorte de plèbe juridictionnelle, hormis les chefs des juridictions des grandes villes. Ils sont surmontés par une élite appartenant aux 27 cours impériales demeurées françaises en 1814 sous le nouveau nom de cours royales ${ }^{7}$ et, audessus encore, par les 55 magistrats de la Cour de Cassation, qui représente le sommet de l'institution judiciaire.

Toutes les générations sont représentées : de jeunes substituts du parquet ou conseillers-auditeurs du siège, âgés de 30 ans à peine, étaient encore dans l'enfance au moment de la Révolution; à l'inverse, les conseillers titulaires, procureurs généraux ou premiers présidents les plus chenus, presque septuagénaires, étaient déjà en pleine maturité en 1789, et ont parfois appartenu aux cours de justice d'Ancien régime, à savoir les Parlements ${ }^{8}$. Plus nombreux encore sont les fils de ces parlementaires, qui ont massivement investi, à leur

5. Étant donné la lenteur de ce renouvellement, les magistrats rapatriés ayant pu se replacer rapidement ont été peu nombreux. On peut toutefois citer comme exemple le procureur général de la cour de Rome, Le Gonidec, qui obtient dès février 1815, une place de conseiller à la Cour de cassation (confirmée en août); son premier avocat général à Rome, Gilbert Boucher, rétrograde comme simple substitut à la cour d'Orléans (octobre 1814), puis de Paris (Cent-Jours), et en février 1816, plus modestement encore, comme procureur au tribunal de Joigny (Yonne) - ces premières affectations lui permettant ensuite de rebondir vers des postes plus prestigieux.

6. En application d'une ordonnance du 29 novembre 1826. Le chiffre global de 1827, à hauteur de 7300 , comprend des non-magistrats (juges de paix et greffiers). Cf. Jean-Claude Farcy, Les carrières des magistrats $\left(X I X^{e}-X X^{e}\right.$ siècles). Annuaire rétrospectif de la magistrature, Centre Georges Chevrier, Université de Bourgogne, rapport de juin 2009, p. 13.

7. Soit au siège, et par ordre hiérarchique, les conseillers-auditeurs (stagiaires), conseillers, présidents de chambre et le premier président; pour le parquet, les juges-auditeurs, substituts, avocats généraux et le procureur général.

8. C'est ainsi que le premier avocat général à la cour d'Aix, d'Eymar de Montmeyan, nommé par Napoléon, exerçait déjà les mêmes fonctions au parlement de Provence avant 1789. 
tour, la magistrature, bien souvent dès le règne de Napoléon qui recherche l'appui de ces familles anciennes de serviteurs de l'État'.

La Restauration des Bourbons à partir d'avril 1814 engendre un processus paradoxal : d'un côté, commence le spectacle apparent d'une unanimité de façade, qui voit se rallier au nouveau régime l'ensemble des corps constitués dans un torrent de flagornerie, suivant une rhétorique de l'éloge tout droit reprise de l'Empire ${ }^{10}$; d'un autre côté, on voit affleurer, à mots couverts, d'anciennes et profondes dissensions qui ressurgissent, liées aux affinités politiques. La magistrature n'échappe pas à ce double mouvement : car, si elle a, plus qu'aucun autre corps sans doute, le souci d'incarner des valeurs telles que la dignité et l'honorabilité, sa tradition ancienne d'indépendance et de politisation la prédispose aux escarmouches frondeuses, dans une sorte d'émulation avec le barreau qu'elle côtoie. Sous la Restauration, le modèle du parlementaire d'Ancien régime en rébellion ouverte contre le «despotisme gouvernemental» du triumvirat formé par Maupeou, Terray et d'Aiguillon (1771-1774) refait surface dans l'imaginaire collectif.

À la fin de l'hiver, c'est, du reste, un ancien magistrat qui donne le signal du basculement contestataire contre l'Empire, à un moment où celui-ci, quoique vacillant, paraît toujours redoutable. Trois semaines avant Paris, Bordeaux décide le 12 mars 1814, par le truchement de ses autorités municipales, d'appeler à la déchéance de Napoléon et à la montée sur le trône de Louis XVIII. Or, l'initiateur de cette journée mémorable, l'édile Jean-Baptiste Lynch, fait précisément partie du groupe d'ex-parlementaires qui animait la justice bordelaise avant 1789 : c'est cette dernière qualité qui est significativement mise en avant par le Journal des débats trois semaines plus tard, en indiquant "que la Providence avait choisi pour donner le signal M. Lynch, ancien magistrat au parlement de Bordeaux " ${ }^{11}$. Il faut dire que Lynch s'était, à l'époque, confronté, comme ses collègues, à l'autoritarisme du chancelier Maupeou, subissant, avec le reste du corps, trois ans de retraite forcée jusqu'à l'avènement de Louis XVI en $1774^{12}$. Quarante ans plus tard, comme pour ouvrir une phase de profondes discontinuités, l'Histoire semble donc bégayer, même si d'autres éléments d'explication interviennent désormais ${ }^{13}$.

9. Ainsi, le jeune Philippe-Louis Meyronnet de Saint-Marc, fils d'un conseiller au parlement de Provence, suit les pas de son père à la cour d'Aix comme substitut dès l'Empire puis avocat général à partir du 28 novembre 1814. De même, et à un degré hiérarchique beaucoup plus élevé, le premier président de la cour d'Amiens Robert Armand Le Sens de Folleville, en poste dès l'Empire et se maintenant durant les Cent-Jours, était le fils d'un procureur général près la cour des comptes de Normandie. On pourrait multiplier à l'infini les exemples.

10. On se rapproche parfois de la veine littéraire et poétique étudiée par Corinne Legoy, dans L'enthousiasme désenchanté : éloge du pouvoir sous la Restauration, Paris, Société des études robespierristes, 2010.

11. Journal des débats politiques et littéraires, samedi 2 avril 1814, p. 1.

12. Lors de la nouvelle crise de 1788 entre Parlements et gouvernement, Lynch joua un rôle beaucoup plus modérateur.

13. En particulier le marasme économique des ports atlantiques, dont l'ex-avocat bordelais et président du corps législatif Joachim Lainé, avait osé se faire l'écho fin 1813 dans un fameux rapport. 
Après cette leçon de courage donnée par l'un de leurs devanciers, certains magistrats en poste brûlent à leur tour d'imiter cette audace pionnière et d'incarner, comme au temps jadis, la voix de la liberté renaissante. C'est ainsi qu'il faut interpréter l'acte d'autorité d'Emmanuel Lebeau, conseiller à la cour impériale de Paris. Le $1^{\text {er }}$ avril 1814, il prend sur lui de convoquer le conseil général de la Seine dont il est le président, et de peser de tout son poids pour faire adopter, par cette institution, une proclamation, plus violente encore que celle de Lynch $^{14}$, préalablement rédigée par l'avocat Nicolas Bellart ${ }^{15}$. Certes, de même que Lynch agissait sous le couvert des troupes anglaises, de même Lebeau bénéficie-t-il de quelques garde-fous, tels que la présence des troupes alliées dans la capitale depuis la veille, ou encore le soutien préalable apporté par le conseiller de préfecture Edme Gauthier d'Hauteserve Despréaux, qui n'est autre que l'oncle maternel du préfet de police Étienne Pasquier ${ }^{16}$ - laissant présager le basculement imminent des forces répressives du régime impérial en faveur de la Restauration. Une telle proclamation officielle, endossée par une autorité publique majeure de la capitale, a contribué, en partie, à faire sortir le Sénat de sa torpeur et à lui faire voter, à son tour, la déchéance de Napoléon, au lendemain de ce coup d'audace.

L'installation du nouveau régime force ensuite l'ensemble des magistrats à prendre collectivement position, au niveau de chaque juridiction, par des adresses de ralliement.

Hiérarchie oblige, c'est à la Cour de cassation qu'il revient de se plier, la première, à un tel exercice. En raison même de sa prééminence, la Cour compte plusieurs anciennes figures de la Révolution. Six conventionnels régicides en font notamment partie, à commencer par le procureur général, chef du Parquet, Merlin de Douai ${ }^{17}$; deux de ses avocats généraux, Pons de Verdun et Thuriot - rapporteur en 1804 du procès Moreau-Cadoudal ${ }^{18}$-, avaient également voté la mort de Louis XVI en 1793, de même que trois conseillers de la cour, Genevois, Oudot et François Lamarque. L'adresse rédigée le 3 avril tente bien de masquer cette composition pour le moins hétérogène de la Cour, par un morceau de rhétorique idoine. Le texte appelle «après plus de vingt ans d'orages et de malheurs, [à] retrouver enfin le repos à l'ombre de ce

14. La proclamation du conseil général de la Seine est intégralement reproduite dans le Journal des débats du 2 avril 1814, p. 3-4.

15. Cf. notice «Bellart» dans Hervé Robert, Philippe Bertholet et Frédéric Ottaviano, Dictionnaire des avocats $d u$ barreau de Paris en 1811, après le rétablissement des barreaux par Napoléon $1^{e r}$, Paris, Riveneuve, 2011, vol. 1, p. 99-109.

16. $C f$. sur le site Geneanet, les données généalogiques établies par Guillaume de Tournemire, http://gw.geneanet.org/pierfit?lang=fr; $\mathrm{p}=\mathrm{edme}+\mathrm{jean} ; \mathrm{n}=$ gauthier $+\mathrm{d}+$ hauteserve.

17. $C f$. sur ce célèbre personnage de la Révolution, régicide et rapporteur de la loi sur les suspects, l'étude d'Hervé Leuwers, Merlin de Douai : un juriste en politique, Arras, APU, 1996.

18. Le vieux chef chouan Cadoudal, lors du procès qui s'acheva par sa condamnation à mort, s'obstina à appeler cet assesseur acharné à sa perte "Tue-Roi", en déformant ironiquement son nom (cf. Jean-Pierre Royer, Histoire de la justice en France, op. cit., p. 479). Cet épisode, vieux seulement de dix ans lors de la Restauration, rendait particulièrement pénible son maintien en poste aux royalistes, jusqu'à l'épuration de février 1815 . 
spectre antique et révéré, qui pendant huit siècles a glorieusement gouverné la France». Bien des juges suprêmes ont du mal à accepter un tel ralliement obséquieux aux Bourbons : le texte n'est initialement adopté que par 33 des 52 juges du siège - parmi lesquels Genevois et le frère de Lazare Carnot -, ainsi que par trois des huit membres du Parquet - dont Pons de Verdun $-{ }^{19}$; le lendemain, douze autres magistrats, prétextant une information tardive, approuvent le ralliement, parmi lesquels Merlin de Douai, Brillat-Savarin ou encore le frère de Sieyès ${ }^{20}$. Un cinquième de la Cour refuse encore de venir à résipiscence, dont Thuriot. Du reste, autant l'adresse précoce du conseil général de la Seine était apparue aussi courageuse que sincère, autant celle de la Cour de cassation, suivant celle du Sénat, apparaît dictée par l'opportunisme et l'instinct de survie, et ne suscite par conséquent aucune reconnaissance particulière de la part des Bourbons : ainsi, 8 des 33 signataires de l'adresse du 3 avril ont été évincés un mois avant le retour de Napoléon aux Cent-Jours, sans égard pour leur ralliement immédiat au régime bourbonien $^{21}$.

Entre temps, la cour impériale de Paris a suivi le mouvement, et pour sa part, elle décide de rédiger non pas une mais deux adresses distinctes, l'une pour le siège, l'autre pour le parquet. Ces deux textes, rédigés le 5 avril 1814, ont une tonalité différente : si la magistrature assise, emmenée par le premier président Séguier, se complaît dans le dithyrambe, les parquetiers en revanche osent, avec quelque insolence, conditionner leur ralliement à «la garantie d'une constitution qui assure à jamais les droits de la nation, du monarque et des citoyens ${ }^{22}$. Cet antagonisme larvé entre siège et parquet, qui montre ici sa première expression forte à Paris, s'explique par une dépendance plus forte des parquetiers à l'égard du régime impérial, qui a pu y installer ses créatures plus commodément qu'au siège.

Le même jour, le tribunal de première instance de la Seine publie lui aussi son adresse de ralliement, et en appelle nommément à "Louis XVIII", là où les deux cours avaient préféré prudemment rester dans l'ellipse littéraire. Sans doute faut-il y voir une habileté du président Try, qui se démarque clairement du Sénat et cautionne d'emblée la fiction juridique d'une continuité dynastique gommant l'interrègne révolutionnaire et impérial. Le procureur Courtin, plus réservé en son for intérieur - comme le suggère la suite des événements en 1815 -, juge opportun de ne pas faire bande à part, contrairement aux parquetiers de la cour d'appel, et de s'associer, avec ses subordonnés, à cette déclaration ostensiblement légitimiste.

19. Journal des débats, 5 avril 1814 , p. 3.

20. Journal des débats, 6 avril 1814, p. 1.

21. Il s'agit de Genevois et Van Toulon, écartés dès 1814, le second comme étranger au territoire français désormais restreint, ainsi que d'Avemann, Bouchau, Delacoste, Dutocq, Oudart et Pons de Verdun, tous les six évincés lors de l'épuration du 15 février 1815 de la Cour de cassation.

22. Journal des débats, 7 avril 1814, p. 2. 
Derrière la courtoisie réciproque affichée, le nouveau pouvoir monarchique n'est pas dupe de ces politesses et certaines premières mesures sonnent déjà comme une mise en garde. Pour preuve, quelques jours avant l'arrivée de Louis XVIII à Paris, son frère Monsieur, en qualité de lieutenant général, décide de valoriser ostensiblement Lecourbe, un ancien conseiller à la cour de Paris jadis destitué comme "prévaricateur» par Napoléon pour sa ferme impartialité lors du procès Moreau-Cadoudal, en lui donnant le titre de "conseiller honoraire» (26 avril 1814 $)^{23}$ : cette semi-réintégration, qui souligne une volonté politique de renouer la chaîne des temps, même dans le domaine juridictionnel, esquisse la tentation d'une recomposition plus vaste, potentiellement menaçante pour d'autres magistrats.

\section{Un DIVORCE CONSOMMÉ AVANT MÊME LE RETOUR DE NAPOLÉON}

À la suite de multiples péripéties qu’on ne peut développer ici, la méfiance entre pouvoir politique et magistrature se renforce au fil des mois. En témoignent, à côté de certaines polémiques dans les journaux, les discussions tendues au Palais-Bourbon à l'été 1814 sur l'inamovibilité des juges, puis à la fin de la même année, les débats animés autour du projet de loi de réduction du personnel de la Cour de cassation ${ }^{24}$.

La réorganisation de la Cour de cassation du 15 février $1815^{25}$, qui en découle, montre les limites de la politique de faux-semblant menée depuis le retour de Louis XVIII, et la volonté des Bourbons d'intimider, depuis le sommet de l'appareil judiciaire, un large pan de la magistrature française resté, au fond, hostile au nouvel ordre des choses et prêt à l'entraver par tous moyens, comme au meilleur temps des luttes parlementaires de l'Ancien régime. La vertu de l'exemple venu d'en haut est censée impressionner les juridictions inférieures, à Paris comme en province. Sont en particulier visés quantité de juges promus sous la Convention ou, plus souvent, sous le Directoire, dont une partie a été maintenue en poste ou réintégrée par Napoléon, par inertie ou souci d'équilibre, et que l'on soupçonne, souvent à raison, d'avoir gardé des nostalgies républicaines; la lenteur de telles instructions, la tiédeur de tels attendus, le laxisme de tels verdicts, y compris dans la justice de droit commun, alimentent durablement à leur endroit le soupçon de sabotage de l'ordre établi, régulièrement dénoncé ensuite par les rapports des procureurs généraux adressés au ministre de la Justice.

23. Journal des débats, 29 avril 1814, p. 3.

24. Cf. Jean-Pierre Royer, «Les épurations judiciaires de 1789 à 1815 », dans Association française pour l'histoire de la justice, L'épuration de la magistrature de la Révolution à la Libération, colloque 4-5 décembre 1992, Paris, Loysel, 1994, p. 30-31.

25. Bulletin des Lois, $5^{\mathrm{e}}$ série, tome 3 , avril 1815 , bulletin $\mathrm{n}^{\circ} 78$, ordonnance du Roi $\mathrm{n}^{\circ} 683$ "contenant Institution des membres composant la Cour de cassation", p. 85-88. Sont écartés le procureur général et deux avocats généraux - dont Pons de Verdun, déjà démissionnaire -, le premier président et huit conseillers. 
Trois semaines après l'épuration de la Cour de cassation, l'ordonnance majeure du 3 mars 1815 prévoit que l'ensemble des cours et tribunaux sera pareillement soumis à ce mécanisme de l'institution royale permettant d'évincer les indésirables : car ce processus, qui confère une présomption légale de capacité au personnel des juridictions concernées, s'accompagne d'une libre recomposition de celles-ci, au bon plaisir du souverain et de son gouvernement ${ }^{26}$. Ainsi, le principe même d'une épuration judiciaire à portée générale est déjà minutieusement décidé et avalisé, alors que le débarquement de Napoléon n'est pas encore connu. Les Cent-Jours et les excès qui les accompagnent affermissent cette volonté; mais celle-ci préexiste au retour de l'Empereur.

Ce déclenchement des hostilités au moment même où Napoléon effectue son retour jette logiquement une grande partie des magistrats dans les bras de ce dernier, et explique qu'un grand nombre n'ait pas refusé de servir l'Empereur, quels que soient leurs sentiments personnels. On peut même se demander si le premier président de la Cour de cassation Muraire, évincé par Louis XVIII le 15 février pour malversation financière, n'a pas activé avec zèle les réseaux de la franc-maçonnerie dont il est un des hauts dignitaires nationaux ${ }^{27}$ afin de faciliter et d'accélérer cette transition judiciaire à tous les niveaux des juridictions.

L'éviction de régicides tels que Merlin de Douai, imposée par la famille royale, apparaissait naturelle et fut, somme toute, trop tardive, même si elle constitue l'acquis principal de la réorganisation de février 1815. Mais le choix des nouveaux promus à la Cour de cassation reste marqué du sceau de l'ambigüité, à l'image de maintes décisions du premier gouvernement Talleyrand et donne un signal équivoque, résultant d'un âpre marchandage entre réactionnaires et libéraux.

Au terme d'une active campagne de presse commencée dès les premiers débuts de la première Restauration, les royalistes "purs", proches de Monsieur, sont parvenus à imposer leur candidat comme chef de la juridiction suprême : de Sèze, l'ancien avocat de Louis XVI, est directement propulsé du barreau à la première présidence de la Cour; après un retrait logique aux Cent-Jours, il conserve ensuite cette éminente fonction jusqu'à sa mort en 1828, parallèlement à une élévation à la pairie qu'il obtient dès le 17 août 1815.

À l'inverse, au parquet, l'un des nouveaux avocats généraux nommés en février 1815, Fréteau de Pény, issu de la cour royale de Paris, s'empresse de trahir Louis XVIII en ralliant le régime napoléonien, à l'instar des bannis du

26. Bulletin des Lois..., op. cit., $\mathrm{n}^{\circ} 85$, ordonnance du Roi $\mathrm{n}^{\circ} 746$ «concernant les Provisions à délivrer aux membres des Cours et tribunaux, et le Tarif du droit de sceau et des droits des Référendaires sur ces provisions», p. 189-191. L'ordonnance évoque «l'installation qui sera faite, d'après nos ordres, de chacune de nos cours et tribunaux, après que nous en aurons nommé et institué les membres".

27. Jean-Louis Debré, La Justice au XIXe siècle, les magistrats, Paris, Perrin, 1981, p. 136. 
15 février qu'il remplace - au passage, on constate que Napoléon, tout en déclarant vouloir strictement revenir à la composition des juridictions antérieure à sa première abdication ${ }^{28}$, est fortement tenté, à son tour, de ménager une partie des promus de la Restauration et donc de prendre en compte la discontinuité de 1814 pour composer un nouvel équilibre ${ }^{29}$. Mais comment s'explique, précisément, semblable promotion par les Bourbons d'un impétrant aussi peu fiable? Le rôle des «illustrations familiales» s'avère souvent considérable, dès l'Empire et plus encore sous la Restauration, en vertu du poids du passé. En l'espèce, même s'il est difficile de sonder les reins et les cœurs, on peut penser que la promotion de Fréteau de Pény a été motivée, en partie, par le souvenir libéral qu'avait laissé son père, Fréteau de Saint-Just, parlementaire frondeur avant 1789, puis Constituant enthousiaste, deux fois président de l'assemblée, à l'initiative de la titulature de «roi des Français» décernée à Louis XVI, et finalement guillotiné sous la Terreur ${ }^{30}$. Dans l'esprit du gouvernement Talleyrand, le fils se mettrait dans les pas du père; sous la houlette modérée de Mourre, renommé procureur général en février 1815 après avoir déjà exercé ces fonctions entre 1800 et 1811, il pourrait faire partiellement contrepoids aux purs comme de Sèze. C'est dans la même logique que le 3 mars 1815, l'ancien député girondin Henri Larivière est nommé, lui aussi, comme avocat général auprès de la Cour de cassation ${ }^{31}$.

Les régicides, jacobins farouches ou bonapartistes trop zélés laissent donc place à un attelage composite de libéraux, incarnation du volet modéré de la Révolution, et de royalistes intransigeants. Le ministre de la Justice Dambray, réactionnaire dans l'âme mais en même temps attaché aux intérêts du roi Louis XVIII, a pu prêter la main à de tels marchandages : il s'agissait de ne pas se laisser totalement circonvenir par les candidats de Monsieur et de rester fidèle à la politique d'équilibre relatif souhaité par le monarque; en outre, les personnalités acceptées comme contrepoids modéré, voire libéral, à l'invitation pressante du clan Talleyrand, étaient prioritairement placées au parquet, c'est-à-dire dans des postes librement révocables, non protégés par le principe d'inamovibilité.

Lors du second retour des Bourbons, après la grande trahison des CentJours par l'essentiel des corps constitués, l'état d'esprit change naturellement

28. Bulletin des Lois, $6^{e}$ série, bulletin $\mathrm{n}^{\circ} 2$, décret $\mathrm{n}^{\circ} 16$ publié à Lyon, 13 mars 1815 , "qui annulle [sic] tous les changemens opérés dans les Cours et tribunaux».

29. Ainsi, le maintien de Fréteau de Pény est annoncé par le Journal des débats du 2 avril 1815, p. 2. Un mois plus tard, le Journal de Paris annonce toutefois son remplacement par Frédéric Bourguignon ( $\mathrm{n}^{\circ} 121,1^{\text {er }}$ mai 1815 , p. 1), mais ce changement n'est, semble-t-il, pas entré en vigueur.

30. Son dossier personnel de carrière (Arch. nat., BB6(II)/165), limité à 4 courriers lors de sa tardive retraite en 1852, ne permet pas de confirmer l'hypothèse. Mais fin 1815, la filiation est explicitement rappelée et soulignée dans Biographie moderne [...] contenant les portraits politiques des Français de l'un et l'autre sexe, morts ou vivants, qui se sont rendus plus ou moins célèbres depuis le commencement de la révolution jusqu’à nos jours, Paris, A. Eymery, 1815, vol. 2, p. 77.

31. Il est confirmé à cette fonction le 28 août 1815 , Arch. nat., BB6*/524/2 (registre de nomination). 
et de telles transactions n'ont plus lieu d'être. L'heure n'est plus à l'équilibre mais bien à la vengeance, et au remplacement de personnalités fortement compromises pendant les Cent-Jours. Le 14 août 1815, une ordonnance est édictée dans cet esprit, qui révoque et remplace le procureur général près la cour d'Amiens, le premier président de la cour de Montpellier, et les deux chefs de juridiction, siège et parquet simultanément, à Toulouse et à Dijon $^{32}$. En révoquant ainsi d'un seul coup six sommités du corps judiciaire, Louis XVIII et son garde des Sceaux modéré, Pasquier, entendent faire un exemple dans l'urgence, et décourager ainsi toute prise de position séditieuse qui remettrait en question la seconde Restauration. Ces évictions individuelles constituent aussi le prélude d'une épuration véritable, qui suppose une réorganisation globale, systémique, de l'ensemble des juridictions, et de ce fait, l'irruption d'une discontinuité beaucoup plus profonde. Promise dès le 3 mars 1815, quelles furent les modalités, mais aussi l'ampleur, d'une telle épuration judiciaire?

\section{LES LIMITES DE L'ÉPURATION JUDICIAIRE}

Certains juristes, proches des ultras, ont encouragé le processus par leurs écrits. Ainsi, Geoffroy Grouard, avocat d'origine berrichonne, donne crânement le ton de son soutien total à la Terreur blanche en août 1815, par des amalgames historiques tranchants : «Les Croisés défendaient la loi de leurs pères en faisant la guerre aux infidèles $»^{33}$. Un mois plus tard, il récidive par un nouveau pamphlet, et vise principalement, cette fois, les juges, reproduisant ainsi la vieille haine corporatiste des avocats à l'égard des magistrats; dans ce texte, la stricte épuration judiciaire se trouve justifiée par la déchéance morale dans laquelle est tombé le corps depuis 1789 :

"Cet ordre si brillant avant la révolution était tombé dans une telle obscurité!!! Quel respect, quelle garantie pouvaient offrir des Magistrats colorés, qui, tous les jours, fournissaient l'occasion d'appliquer cette censure amère d'un poëte comique [Beaumarchais], qui fait dire à Bridoison : Est-ce que j’ai acheté ma charge pour autre chose? ${ }^{34}$

Approuvant tacitement la remise en ordre impériale, Grouard en appelle désormais à une nouvelle épuration, dans un sens royaliste, pour parachever le travail : «La gravité du mal a déjà commandé une première épuration;

32. Ordonnance reproduite dans Jean-Gabriel Peltier, L'Ambigu, ou Variétés littéraires et politiques, Londres, Schulze et Dean, vol. 50, 1815, p. 612-613.

33. G. Y. Grouard, docteur en droit, Lettre au Roi sur la situation intérieure de la France, Paris, Testu, $2^{e}$ éd. revue et augmentée, août 1815, p. 12.

34. G. Y. Grouard, Du Législateur, du Magistrat et du Citoyen, d'après la Charte constitutionnelle, Paris, Testu, septembre 1815, p. 81-82. 
l'organisation définitive sera telle qu'on est en droit de l'attendre d'un gouvernement légitime, ami des mœurs et protecteur de la sécurité publique». Influencé par l'agressivité de l'époque, il évoque même avec gourmandise les coutumes terrifiantes de la Grèce ancienne : «Une loi de Minos permettait à tout citoyen de tuer un Magistrat qu'il trouvait ivre. Solon porta une loi semblable ${ }^{35}$. On voit ici à quel point les haines politiques se greffent à l'intérieur même du monde judiciaire pour stimuler l'esprit de revanche et l'appel ouvert aux règlements de comptes, expressément destinés aux magistrats progressistes, ayant trop longtemps abusé de leur position dominante depuis 1789. Mais sur une épuration de quelle ampleur débouchent, au juste, de tels appels?

Les ouvrages de référence sur l'histoire de la magistrature donnent toujours les mêmes chiffres. 1400 ou 1694 magistrats sont censés avoir été épurés des tribunaux de première instance; les deux chiffrages sont donnés concurremment, sans explication du fort différentiel de $20 \%{ }^{36}$; il est vrai que le grand nombre des juridictions et surtout l'étalement de l'épuration, sur près de six ans $s^{37}$, rendent ce dénombrement particulièrement complexe. En revanche, l'historiographie tombe unanimement d'accord sur le degré d'épuration pour le niveau supérieur des cours royales : 294 magistrats auraient été épurés entre l'été 1815 et l'année 1818. Ce chiffrage repose sur l'addition des chiffres donnés, pour 19 des 27 cours, en 1818, par Alphonse Bérenger (1785-1866), magistrat libéral fraîchement épuré, dans un ouvrage de circonstance ${ }^{38}$. Il est vrai que l'auteur à l'origine de cette vulgate chiffrée affichait une assurance à toute épreuve, qui porta visiblement ces fruits : « $\mathrm{Si}$ ce tableau renferme quelques inexactitudes, elles porteraient sur un ou deux magistrats au plus, qu'il faudrait ou retrancher, ou ajouter à l'épuration de chaque cour $»^{39}$. Est-ce si sûr?

L'examen de la cour royale de Paris, qui fut chronologiquement, la première à être instituée et donc fixée sur l'identité de ses membres, et pour laquelle Bérenger dénombre 29 magistrats épurés, montre l'ampleur de la supercherie, qui dépasse de beaucoup une ou deux unités. On note effectivement que 29 magistrats n'occupent plus leur fonction antérieure, à la suite

35. Ibidem, p. 80.

36. Jean-Louis Debré cite sans sourciller les deux chiffres sur la même page (La Justice au XIXe siècle, Les magistrats, op. cit., p. 50); le chiffre de 1694 est déjà avancé par Marcel Rousselet dans Histoire de la magistrature française..., op. cit., vol. 2, p. 164.

37. Le dernier tribunal institué est celui de Tarascon le 27 juin 1821.

38. Alphonse Bérenger, De la justice criminelle en France, d'après les lois permanentes, les lois d'exception et les doctrines des tribunaux, Paris, C-F. Patris, 1818, p. 230-231; à sa suite Georges MartinSarzeaud, Recherches historiques sur l'inamovibilité de la magistrature, 1883, p. 457; Marcel Rousselet, Histoire de la magistrature..., op. cit., 1957, vol. 2, p. 167; Jean-Louis Debré, Les magistrats..., op. cit., 1982, p. 208; Renée Martinage "L'épuration sous les régimes monarchiques" dans L'épuration de la Révolution à la Libération, actes du colloque de l'Association française pour l'histoire de la justice, 4-5 décembre 1992, Paris, Loysel, 1994, p. 37 ; Jean-Pierre Royer, Histoire de la justice en France, op. cit., p. 495; Jean-Claude Farcy arrondit à «près de 300 » dans L'histoire de la justice française..., op. cit., p. 222 et retient le chiffre de 1400 pour les tribunaux de $1^{\text {re }}$ instance.

39. Bérenger, De la justice criminelle en France..., op. cit., p. 231. 
de l'institution du 18 septembre 1815 , ce qui permet de faire de la place à certains royalistes incontestables, à l'image de Bellart, directement propulsé du barreau à l'éminente fonction de procureur général près la cour royale. Mais le décompte précis ne laisse pas d'être édifiant : un magistrat du siège est réintégré quelques semaines à peine après son épuration; onze magistrats $\mathrm{du}$ siège et deux parquetiers connaissent des promotions indiscutables, au sein de la cour de Paris ${ }^{40}$, ou même à la cour de Cassation, ne pouvant, en aucune manière, être assimilées à une sanction. Seuls 15 magistrats sur les 29 sont évincés, dont cinq parquetiers ${ }^{41}$ qui sont, par définition, révocables ad libitum, n'étant pas, depuis l'an VIII, protégés par quelque inamovibilité que ce soit, et deux conseillers-auditeurs, c'est-à-dire des stagiaires embauchés à l'essai, sans aucune garantie de titularisation ${ }^{42}$. Enfin, sur les 48 juges du siège titulaires, seuls 8 (soit $17 \%$ ) sont effectivement et sans abus de langage, épurés, au mépris de leur inamovibilité théorique, garantie par la Charte ${ }^{43} .8$ vrais épurés, au lieu des 29 annoncés.

Au tribunal de la Seine, institué le 15 octobre 1815, 6 juges du siège titulaires sont pareillement épurés sur les 36 en poste un an plus tôt (17\%), tandis qu'un auditeur et six parquetiers sont simultanément évincés, dont le procureur Courtin, qui s'était surpassé, pendant les Cent-Jours, dans l'hostilité aux Bourbons; à l'inverse, 5 juges du siège, 3 auditeurs et 1 substitut bénéficient d'une promotion.

L'épuration a-t-elle été plus sévère en province? L'institution de la cour de Lyon, qui intervient dix jours plus tard, pourrait le laisser penser; ici, ce sont 9 juges du siège titulaires sur $30(30 \%)$ qui subissent l'épuration, parallèlement à 8 promotions réelles, tandis que 6 membres du parquet sont simultanément remerciés ${ }^{44}$. L'implication tapageuse de la cour lyonnaise, premier président en tête, dans l'organisation d'un "pacte fédératif» anti-royaliste durant les Cent-Jours, y est naturellement pour beaucoup ${ }^{45}$. Tandis que la situation parisienne n'avait nécessité qu'une réaction modérée, cette sévérité ciblée, réclamée par le duc d'Angoulême ${ }^{46}$, pouvait faire figure d'avertissement pour les autres juridictions du royaume. La situation des autres cours,

40. Des conseillers-auditeurs sont titularisés, des conseillers deviennent présidents de chambre.

41. Le procureur général Legoux, les avocats généraux Giraudet et Girod de l'Ain, les substituts Paradis et Leloup de Sancy.

42. Ces conseillers-auditeurs sont Poriquet et Merilhou, ce dernier futur dignitaire du parti libéral et ministre de la Justice du gouvernement Laffitte (décembre 1830-mars 1831), à son tour auteur d'une vaste épuration.

43. Il s'agit des présidents Gilbert des Voisins et Nougarède de Fayet, et des conseillers Royer, Desclozeaux, Thomas, Delecroix, Jaubert, Bourguignon-Dumolard.

44. Bérenger annonçait logiquement, quant à lui, 23 «épurations» pour cette cour lyonnaise (idem). Noter que le conseiller Deydier, régicide, avait devancé l'épuration en démissionnant de la cour dès le 8 juin 1814, avant d'être réintégré durant les Cent-Jours, décision évidemment invalide pour le régime bourbonien.

45. Cf. à ce sujet Jean-Marie Vincent Audin, Tableau historique des événemens qui se sont passés à Lyon depuis le retour de Bonaparte jusqu'au rétablissement de Louis XVIII, 2 éd., Lyon, Guyot frères, 1815 .

46. Cf. Laurent Nagy, D’une Terreur à l'autre..., op. cit., p. 18. 
déjà étudiée par certains travaux ${ }^{47}$, est variable, avec une épuration bien plus légère à Douai, par exemple, qu’à Rennes, mais, quel que soit le cas d'espèce, avec des chiffres systématiquement moins importants que ceux transmis par la tradition.

Si l'ampleur de l'épuration judiciaire est donc plus modeste que ce qu'a souligné jusqu'ici l'historiographie, illusionnée par la démonstration factice de Bérenger, la lenteur de ce processus, soulignée par Jean-Pierre Royer, est tout aussi frappante ${ }^{48}$. Au 31 décembre 1815, seules 2 cours royales sur 27, Paris et Lyon, ont été instituées et ont donc subi l'épuration; ce qui signifie que pour les autres cours, l'angoissant suspense a continué, quelques mois supplémentaires pour les 14 premières $^{49}$, et jusqu'en décembre 1818 pour les 11 dernières à être réorganisées ${ }^{50}$, dans un tout autre contexte, marqué par une réorganisation de la carte judiciaire ${ }^{51}$ - ce qui a donné à ces derniers mouvements une coloration nettement moins politique et plus technique, aspect souvent négligé. La véritable efficacité de l'épuration, beaucoup plus que dans son ampleur, a donc tenu à ces délais : car dans l'attente d'une institution royale qui les confirme à leur poste, les juges du siège ont été conduits à adopter une position servile à l'égard des desiderata du gouvernement. Dans ce laps de temps volontairement prolongé, leur traditionnel surcroît d'indépendance par rapport aux parquetiers s'est donc assez fortement réduit.

Néanmoins, les membres du Parquet restaient bien soumis à la précarité la plus forte, car eux pouvaient être démis à n'importe quel moment, indépendamment de ce calendrier officiel de l'épuration légale, qui ne les concernait pas et ne les mettait d'ailleurs pas à l'abri, une fois l'institution royale de la juridiction obtenue. Ils ont donc été, plus que tout autre, astreints à un légitimisme ostentatoire, sinon à une obséquieuse prudence dont rendent compte maints discours de rentrée prononcés chaque début du mois de novembre ${ }^{52}$.

En vérité, même pour les épurés du siège ou les évincés du parquet, il s'est présenté, dès 1817, des occasions de repêchage avidement saisies par une partie très significative des relégués - hors extrémistes avérés ou régicides relaps,

47. Didier Veillon, Magistrats au XIX' siècle : en Charente-Maritime, Vienne, Deux-Sèvres et Vendée, La Crêche, Geste, 2001 ; Laurence Soula, La robe, la terre et le code. La cour d'appel d'Agen (18001851), thèse histoire du droit, Université de Toulouse-1, 1996; Chantal Dauvergne, La cour d'appel de Dijon (an VIII-1852), thèse histoire du droit, université de Bourgogne, 1990.

48. Jean-Pierre Royer, Histoire de la justice en France, op. cit., p. 496.

49. Entre le 3 janvier et le 26 avril 1816 pour les cours de Rennes, Bordeaux, Dijon, Orléans, Aix, Metz, Nancy, Ajaccio, Pau, Grenoble, Toulouse, Besançon, Colmar et Douai.

50. Le 8 décembre pour les cours d'Agen, Amiens, Angers, Caen, Limoges, Montpellier, Nîmes et Riom, et le 17 décembre pour celles de Bourges, Poitiers et Rouen. Cf. Arch. nat., BB6*/524/2, registre de nomination.

51. Cf. Bulletin des Lois, $7^{\text {e }}$ série, t. 7, ordonnance du 8 novembre 1818 n 5556 , p. 766 , avec création d'une quatrième chambre à la cour de Toulouse et la réduction simultanée d'une chambre à Agen, Angers et Amiens, en raison du volume d'affaires traitées par an.

52. Cf. à ce sujet l'étude de Jean-Claude Farcy, Magistrats en majesté. Les discours de rentrée aux audiences solennelles des cours d'appel (XIX'e-XXe siècles), Rapport de recherche de l'université de Paris X-Nanterre, juillet 1996, 2 vol. et Paris, CNRS éditions, 1998, 793 p. 
naturellement. Cette réintégration s'observe à tous les niveaux de l'appareil judiciaire. Prenons deux exemples significatifs.

En province, Nicolas Boyard, ancien avocat au barreau de Paris, était devenu, par la grâce de la Restauration, conseiller-auditeur à la cour royale d'Orléans le 5 décembre 1814, et n'avait pas jugé utile de démissionner expressément lors des Cent-Jours. Indice de l'amateurisme qui caractérise la vague d'épuration sous la "Terreur blanche", il est d'abord promu au parquet comme substitut de la même cour le 23 septembre 1815, avant d'être évincé lors de la réorganisation de la compagnie en février 1816. Réflexion faite, il est réintégré comme substitut à la cour de Nancy dès le 26 mars 1817, avant d'être promu, par la droite elle-même, avocat général (octobre 1820) puis conseiller (octobre 1821), jusqu'à sa promotion comme président de chambre à Orléans en 1833 par le régime de Juillet ${ }^{53}$.

Les plus hautes juridictions parisiennes connaissent, elles aussi, un tel mouvement de contre-épuration, motivé par la nécessité de s'appuyer sur les talents authentiques pour faire correctement fonctionner l'institution judiciaire. Pour reprendre jusqu'au terme de la démonstration, l'exemple particulièrement net de Fréteau de Pény, celui-ci, logiquement épuré au début de la seconde Restauration ${ }^{54}$ pour prix de sa trahison des Cent-Jours, est solennellement réintégré à son poste d'avocat général près la Cour de cassation dès 1818 , lors d'une cérémonie assez théâtrale qui permet au premier président de la juridiction suprême, l'ultra-royaliste de Sèze, de se poser en paternaliste donneur de leçons vis-à-vis de tous les magistrats tentés par la désobéissance ${ }^{55}$. Une telle dramaturgie du pardon conditionnel, bruyamment réintroduite pendant l'ère Decazes pour tourner la page de la «Terreur blanche», est, du reste, d'une efficacité limitée, comme en témoigne la nouvelle révocation de l'intéressé par Peyronnet en août 1824 - au grand dam du président de Sèze, hostile à cette sanction $-^{56}$, pour avoir pris, cette fois, des réquisitions trop clémentes à l'égard de L'Aristarque français, le journal d'extrême-droite lancé par La Bourdonnaye. Cet épisode postérieur est la preuve qu'un tel magistrat, archétypal à bien des égards, n'est pas seulement une "girouette" indifférente à l'égard des régimes qu'il sert, mais surtout un homme de loi foncièrement attaché à son indépendance, suffisamment compétent pour être périodiquement réintégré par les pouvoirs successifs jusqu’à la monarchie de Juillet, qui finit par en faire un pair de France en 1835.

*

53. $C f$. Arch. nat., $\mathrm{BB}^{*} 530, \mathrm{n}^{\circ} 4407$ (fiche matricule), et Hervé Robert et alii, Dictionnaire des avocats..., op. cit., vol. 1, p. 200-204.

54. Bulletin des Lois, $7^{\mathrm{e}}$ série, tome 1 , février 1816, bulletin $\mathrm{n}^{\circ} 19$, ordonnance du Roi $\mathrm{n}^{\circ} 89 \mathrm{du}$ 28 août 1815. Fréteau est remplacé par Cahier, auparavant simple substitut à la cour royale de Paris.

55. Germain Sarrut et B. Saint-Edme, Le Plutarque de 1847: biographie des hommes du jour, Paris, Revue de Savoie, 1847 [1 ${ }^{\text {re }}$ éd. 1835-1842], p. 101.

56. Le Provincial, recueil périodique, Dijon, jeudi 8 mai 1828, p. 18. 
L'épuration de la magistrature, mûrement décidée avant même les CentJours, s'est révélée d'autant plus efficace qu'elle fut sélective, progressive dans son déroulement et à l'inverse rapidement réversible pour la plupart des magistrats de bonne volonté acceptant de rentrer dans le rang. Néanmoins, l'idéal d'indépendance continue à séduire une large fraction de la magistrature, y compris dans les rangs royalistes : c'est précisément parce que l'épuration judiciaire de la seconde Restauration n'a pas été aussi massive et brutale qu'on l'a dit, que ces rapports dialectiques entre politique et magistrature ont conservé toute leur actualité bien après 1815 , alimentant périodiquement la chronique des règnes de Louis XVIII et de Charles X.

Olivier Tort est maître de conférences à l'Université d'Artois 